\title{
QTL mapping for fiber quality and yield- related traits across multiple generations in segregating population of CCRI 70
}

\author{
DENG Xiaoying ${ }^{1,2 \dagger}$, GONG Juwu ${ }^{1 \dagger}$, LIU Aiying ${ }^{1 \dagger}$, SHI Yuzhen ${ }^{1}$, GONG Wankui', GE Qun, LI Junwen', \\ SHANG Haihong ${ }^{1}$, WU Yuxiang ${ }^{2^{*}}$ and YUAN Youlu ${ }^{1 *}$
}

\begin{abstract}
Background: Cotton is a significant economic crop that plays an indispensable role in many domains. Gossypium hirsutum L. is the most important fiber crop worldwide and contributes to more than $95 \%$ of global cotton production. Identifying stable quantitative trait locus (QTLS) controlling fiber quality and yield related traits are necessary prerequisites for marker-assisted selection (MAS).
\end{abstract}

Results: A genetic linkage map was constructed with 312 simple sequence repeat (SSR) loci and 35 linkage groups using JoinMap 4.0; the map spanned 1929.9 cM, with an average interval between two markers of $6.19 \mathrm{cM}$, and covered approximately $43.37 \%$ of the cotton genome. A total of 74 QTLs controlling fiber quality and 41 QTLs controlling yield-related traits were identified in 4 segregating generations. These QTLs were distributed across 20 chromosomes and collectively explained $1.01 \% \sim 27.80 \%$ of the observed phenotypic variations. In particular, 35 stable QTLs could be identified in multiple generations, 25 common QTLs were consistent with those in previous studies, and 15 QTL clusters were found in 11 chromosome segments.

Conclusion: These studies provide a theoretical basis for improving cotton yield and fiber quality for molecular marker-assisted selection.

Keywords: QTL mapping, Fiber quality, Yield quality, Multiple generations, Upland cotton (Gossypium hirsutum L.)

\section{Background}

Cotton is an important cash crop, and its fiber is the most important renewable natural resource for the textile industry. Upland cotton (Gossypium hirsutum L.) is the most important cotton species, accounting for more than 95\% of cotton production worldwide (Chen et al. 2008; Lacape et al. 2003; Shang et al. 2015). Improving fiber quality while maintaining a high yield potential of Upland cotton is an important research direction in cotton breeding. Because of the negative correlation between yield and fiber quality traits (Rong et al. 2004; Shen et al. 2005;

\footnotetext{
*Correspondence: yuxiangwu2009@hotmail.com; yuanyoulu@caas.cn

${ }^{\dagger}$ Deng Xiaoying, Gong Juwu and Liu Aiying contributed equally to this work. ${ }^{2}$ College of Agriculture, Shanxi Agricultural University, Taigu 030801, Shanxi, China

${ }^{1}$ State Key Laboratory of Cotton Biology, Key Laboratory of Biological and Genetic Breeding of Cotton, Ministry of Agriculture and Rural Affairs/Institute of Cotton Research, Chinese Academy of Agricultural Sciences, Anyang 455000, Henan, China
}

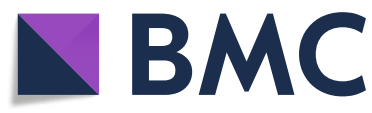

(C) The Author(s). 2019 Open Access This article is distributed under the terms of the Creative Commons Attribution 4.0 International License (http://creativecommons.org/licenses/by/4.0/), which permits unrestricted use, distribution, and reproduction in any medium, provided you give appropriate credit to the original author(s) and the source, provide a link to the Creative Commons license, and indicate if changes were made. The Creative Commons Public Domain Dedication waiver (http://creativecommons.org/publicdomain/zero/1.0/) applies to the data made available in this article, unless otherwise stated. multiple traits in cotton breeding. Although conventional breeding has played a vital role in the genetic improvement of fiber quality and yield-traits in Upland cotton, the achievements and progress have been slow (Zhang et al. 2009). With the development of molecular marker technology, through the construction of saturated genetic map, molecular markers tightly linked to yield and fiber quality can be used to pyramid target genes for the simultaneous improvement of fiber quality and yield potential.

Identification of stable quantitative trait loci (QTLs) is essential for breeding cotton cultivars with superior quality and high yield. Many reports on mapping qualitative traits have involved interspecific populations (Kumar et al. 2012; Lacape et al. 2013; Yu et al. 2013; Zhang et al. 2012, 2013, Said et al. 2015; Zhai et al. 2016; Guo et al. 2018), and intraspecific populations 
(Ademe et al. 2017; Liu et al. 2017; Tang et al. 2015; Shao et al. 2014; Shang et al. 2015; Zhang et al. 2013).

To identify stably expressed QTLs, permanent populations have been used for QTL mapping of fiber quality and yield in recent years (Ademe et al. 2017; Jamshed et al. 2016; Ning et al. 2014; Shen et al. 2007; Shang et al. 2015; Wan et al. 2007). Jamshed et al. (2016) identified one QTL for fiber strength (FS) located on Chromosome 25 by using recombinant inbred lines (RILs), which was the same QTL detected by Sun et al. (2012). This QTL was stably expressed in multiple environments and could be used for MAS (Guo et al. 2005). Constructing multigenerational segregating populations is a highly effective method to identify stable QTLs. Thus, identifying QTLs in early generations of segregating populations would allow us to tag stable QTLs for MAS and accelerate the process of breeding for better fiber quality and higher yield. Therefore, we used hybrid of CCRI 70, a Chinese national approved variety with excellent fiber quality and good fiber yield, to construct $\mathrm{F}_{2}, \mathrm{~F}_{2: 3}, \mathrm{~F}_{2: 4}$ and $F_{2: 5}$ populations for identifying QTLs associated with fiber quality and yield-related traits. The detected stable and common QTLs could be further used to identify the molecular genetic mechanism of fiber quality and yield component traits and in MAS breeding.

\section{Materials and methods}

\section{Plant materials}

The Upland cotton hybrid CCRI $70\left(\mathrm{~F}_{1}\right)$, which comes from the cross between 901-001 $\left(\mathrm{P}_{1}\right)$ and sGK156 $\left(\mathrm{P}_{2}\right.$, as female parent), is a national authorized cotton variety with excellent fiber quality, i.e., an average fiber strength (FS) of $33.5 \mathrm{cN} \cdot$ tex $^{-1}$, fiber length (FL) of $32.5 \mathrm{~mm}$, and fiber micronaire (FM) of 4.3 (Yuan et al. 2009). Line sGK156 is selected from the commercial transgenic cultivar sGK9708 (CCRI 41) that is resistant to cotton Verticillium wilt and cotton bollworm. It has an excellent yield and comprehensive agronomic traits, with an average FM of no more than 4.2. In addition, 901-001 is a line with high fiber quality due to introgression from Gossypium barbadense to Gossypium hirsutum.

Detailed information on this population was provided by Ye et al. (2016). Briefly, an $F_{1}$ combination between sGK156 and 901-001 was made in Anyang, Henan Province, in 2011. $F_{1}$ seeds were sowed in Hainan in the winter of 2011-2012, $F_{2}$ seeds and the two parents were sown in Anyang, Henan Province in 2012, and $250 \mathrm{~F}_{2}$ plants were harvested for fiber quality. The $250 \mathrm{~F}_{2: 3}$ plants were grown in 250 rows that were $5 \mathrm{~m}$ long and $0.8 \mathrm{~m}$ apart in Anyang in 2013, $\mathrm{F}_{2: 4}$ plants were grown in Hainan in the winter of 2013-2014, and $F_{2: 5}$ plants were grown in Anyang in 2014. Thirty naturally opened bolls with two self-crossed bolls were hand-harvested from every plant in the $F_{2: 3}$ to $F_{2: 5}$ generations to generate progeny and test for fiber yield and quality. After the seed cotton samples were weighed and ginned, boll weight (BW) and lint percentage (LP) were evaluated accordingly. The fiber quality traits, including FL, FS, FM, FU and FE, were tested with an HFT9000 using international high-volume instrument calibration cotton (HVICC) samples at the Cotton Quality Supervision and Testing Center of the Ministry of Agriculture of China.

\section{DNA extraction and genotype analysis}

Young leaves were collected from plants labeled $F_{2}, P_{1}$, $\mathrm{P}_{2}$, and $\mathrm{F}_{1}$, frozen in liquid nitrogen and stored at $-80^{\circ} \mathrm{C}$. Genomic DNA was extracted individually as described by Paterson et al. (1993). A total of 14820 simple sequence repeat (SSR) primer pairs were used to screen polymorphisms between parents. The polymorphic primer pairs were used to genotype the $F_{2}$ population. PCR was conducted as described by Sun et al. (2012), and the electrophoresis and detection of PCR products were conducted according to the protocol of Zhang et al. (2000).

A total pool of 14820 pairs of SSR primers were used to screen for polymorphisms between sGK156 and 901-001. The SSR primer sequences were obtained from the following sources: BNL (Brookhaven National Laboratory, NY), HAU (Huazhong Agricultural University, China), NAU (Nanjing Agricultural University, China), STV and CIR (French Agricultural Research Centre for International Development, France), CM and JESPR (Texas A\&M University, USA), DPL and CGR (Delta and Pine Land, USA), SWU and PGML (Southwest university, China), MUCS and MUSS (University of California Davis, USA), Gh and TMB (United States Agricultural Research Service, USA). All of the SSR primer pairs were synthesized by Sangon Biotech (Shanghai, China).

\section{Data analysis}

The genetic map was constructed using JoinMap 4.0 software with a logarithm of odds (LOD) score of 5.0 and a recombination frequency of 0.40 . The Kosambi's mapping function (Kosambi 1994) was used to convert the recombination frequencies into map distances. The linkage groups were drawn by Map Chart 2.2 software (Voorrips 2006). Linkage groups were assigned to corresponding chromosomes according to the chromosomesanchored SSR markers used in previous reports (Lacape et al. 2003, 2013; Rong et al. 2004; Guo et al. 2007; Qin et al. 2008; Xia et al. 2014; Yu et al. 2013; Zhang et al. 2013; Liu et al. 2017; Nie et al. 2016).

QTLs affecting fiber quality and yield-related traits in 4 generations was detected by the composite interval mapping (CIM) method (Zeng, 1994) using Windows QTL Cartographer 2.5 (Wang et al. 2006) with LOD threshold of 2.5 and a mapping step of 1.0 centimorgans 
(cM). QTLs at the same location for the same trait across different generations were regarded as 'stable', and QTLs explaining more than $10 \%$ of the phenotypic variance (PV) were regarded as 'major'. QTL nomenclature was defined as q + traits abbreviation + chromosomes + QTL number (McCouch et al. 1997). In addition, QTL clusters were inferred based on regions containing three or more QTLs for various traits. Regions of approximately $20 \mathrm{cM}$ were taken into account when estimating the presence of a cluster. Clusters were named according to the chromosome on which they were found.

\section{Results}

\section{Phenotypic evaluation of fiber quality and yield traits}

The fiber quality and yield traits phenotype data for the $\mathrm{P}_{1}, \mathrm{P}_{2}, \mathrm{~F}_{2}, \mathrm{~F}_{2: 3}, \mathrm{~F}_{2: 4}$ and $\mathrm{F}_{2: 5}$ populations are presented in Table 1. Skewness and kurtosis values were calculated, and the results indicated that all fiber-related traits showed a normal distribution and transgressive segregation in both directions in the 4 generations (Table 1), indicating that these traits were controlled by multiple genes and suitable for QTL mapping.

\section{Correlation analysis of fiber quality and yield traits in 4 generations}

The correlation coefficients of fiber and yield traits in 4 generations were showed in Table 2. The majority of fiber quality traits were significantly associated with each other, indicating that the genes of different traits were linked and had multiple effects. FL was significantly positively correlated with FS and FU, but was significantly negatively correlated with FM; FS was significantly positively correlated with FU but was negatively correlated with FM (except in the $\mathrm{F}_{2}$ generation). BW was not significantly correlated with most of fiber-related traits (except in the $\mathrm{F}_{2}$ generation). In contrast, LP was significantly negatively correlated with FL, FS and BW but was significantly positive correlated with FM (except in the $F_{2}$ generation).

Correlation analysis between traits in different generations was conducted using the mean value of the four generations (Additional file 1 Table S1). All correlation of FL was significantly positively correlated among generations, and the correlation coefficients among generations varied from 0.150 to 0.348 . Correlation analysis of FS, BW and LP among generations was similar to that for FL. The majority of FM correlation coefficients were significant and positive across generations. The correlation coefficients for FE were more complex, which may relate to environments.

\section{Construction of the genetic map}

Two hundred and sixty-seven of the 14 820 SSR primer pairs $(1.80 \%)$ amplified polymorphisms between two
Table 1 The phenotypic analysis of fiber quality and yieldrelated traits in different generations

\begin{tabular}{|c|c|c|c|c|c|c|c|c|c|}
\hline \multirow[t]{2}{*}{ Trait } & \multirow[t]{2}{*}{ Gen. } & \multicolumn{2}{|l|}{ Parent } & \multicolumn{6}{|c|}{ Population } \\
\hline & & $P_{1}$ & $P_{2}$ & Max. & Min. & Mean & $\mathrm{CV} / \%$ & Skew. & Kurt \\
\hline \multirow[t]{4}{*}{$\mathrm{FL}$} & $\mathrm{F}_{2}$ & 30.54 & 30.77 & 34.84 & 25.35 & 30.44 & 5.27 & -0.19 & -0.04 \\
\hline & $F_{2: 3}$ & 30.41 & 30.24 & 34.08 & 28.89 & 31.17 & 3.26 & 0.39 & 0.01 \\
\hline & $F_{2: 4}$ & 32.23 & 29.85 & 33.67 & .17 & 56 & 3.27 & 0.02 & 0.03 \\
\hline & $F_{2: 5}$ & 32.98 & 31.87 & 35.01 & 29.28 & 32.55 & 3.38 & -0.07 & -0.15 \\
\hline \multirow[t]{4}{*}{ FS } & $\mathrm{F}_{2}$ & 30.01 & 29.54 & 34.60 & 23.40 & 29.95 & 5.09 & -0.04 & 1.04 \\
\hline & $F_{2: 3}$ & 35.15 & 32.63 & 39.10 & 27.20 & 32.43 & 5.53 & 0.36 & 0.48 \\
\hline & $F_{2: 4}$ & 31.20 & 32.90 & 36.50 & 27.40 & 31.35 & 4.7 & 0.2 & 0.6 \\
\hline & $F_{2: 5}$ & 31.90 & 30.73 & 36.40 & 28.60 & 31.87 & 4.16 & 0.45 & 0.39 \\
\hline \multirow[t]{4}{*}{ FM } & $\mathrm{F}_{2}$ & 3.92 & 3.56 & 4.88 & 63 & 3.7 & 13.60 & -0.09 & -0.96 \\
\hline & $F_{2: 3}$ & 4.89 & 3.50 & 5.43 & 3.66 & 4.63 & 6.80 & 0.12 & -0.09 \\
\hline & $F_{2: 4}$ & 3.94 & 3.9 & 4 & .08 & 3.8 & 7.1 & 0.11 & 0.06 \\
\hline & $F_{2: 5}$ & 4.50 & 4.76 & 5.33 & 3.71 & 4.56 & 7.00 & -0.20 & -0.24 \\
\hline \multirow[t]{4}{*}{$\mathrm{FE}$} & $\mathrm{F}_{2}$ & 7.23 & 6.93 & 7.70 & 5.80 & 7.03 & 3.55 & -0.19 & 1.8 \\
\hline & $F_{2: 3}$ & 6.05 & 6.17 & 6.6 & 5.80 & 6.1 & 1.9 & 0.0 & 0.7 \\
\hline & $F_{2: 4}$ & 6.30 & 6.20 & 6.50 & 6.10 & 6.26 & 1.24 & 0.10 & 0.04 \\
\hline & $F_{2: 5}$ & 7.90 & 7 & 8.60 & 7.50 & 8.0 & 2.2 & -0.02 & 0.0 \\
\hline \multirow[t]{4}{*}{ FU } & $\mathrm{F}_{2}$ & 83.41 & 83.58 & 88.50 & 77.90 & 84.29 & 2.10 & -0.68 & 0.55 \\
\hline & $F_{2: 3}$ & 84.55 & 85.07 & 87.30 & 80.80 & 84.90 & 1.18 & -0.46 & 1.12 \\
\hline & $F_{2: 4}$ & 86.20 & 85.30 & 87.80 & 82.40 & 85.35 & 1.07 & -0.23 & -0.03 \\
\hline & $F_{2: 5}$ & 85.10 & 85.57 & 88.30 & 83.80 & 86.11 & 1.03 & -0.21 & -0.27 \\
\hline \multirow[t]{4}{*}{ BW } & $\mathrm{F}_{2}$ & 5.44 & 4.75 & 6.91 & 3.39 & 4.95 & 13.52 & 0.23 & -0.27 \\
\hline & $F_{2: 3}$ & 5.45 & 4.21 & 6.85 & 4.82 & 5.87 & 5.99 & -0.16 & -0.001 \\
\hline & $F_{2: 4}$ & 5.31 & 4.66 & 0.71 & 0.90 & 5.4 & 8.84 & -0.38 & 0 \\
\hline & $F_{2: 5}$ & 5.85 & 4.78 & 7.49 & 5.20 & 6.44 & 5.97 & -0.13 & 0.31 \\
\hline \multirow[t]{4}{*}{ LP } & 12 & 31.04 & 32.90 & 43.12 & 23.96 & 33.11 & 10.20 & -0.11 & -0.25 \\
\hline & $F_{2: 3}$ & 32.51 & 35.28 & 41.39 & 28.61 & 34.57 & 5.70 & 0.16 & 0.34 \\
\hline & $F_{2: 4}$ & 33.65 & 35.11 & 49.28 & 37.64 & 42.31 & 4.30 & 0.17 & 0.32 \\
\hline & $F_{2: 5}$ & 36.63 & 43.44 & 43.04 & 32.29 & 36.52 & 5.40 & 0.36 & 0.16 \\
\hline
\end{tabular}

$F L$ Fiber length $(\mathrm{mm})$, FS Fiber strength $\left(\mathrm{cN}^{\mathrm{N}} \mathrm{tex}^{-1}\right), F M$ micronaire, $F E$ Fiber elongation(\%), FU Fiber uniformity(\%), BW Boll weight(g), LP Lint percentage(\%).

parents. A total of 342 loci were obtained from amplification of the 267 SSR primer pairs in the $250 \mathrm{~F}_{2}$ individuals. After linkage analysis of all 342 polymorphic loci, 312 were mapped to 35 linkage groups (Fig. 1 and Additional file 8 Table S8), thus covering $1929.9 \mathrm{cM}$ with an average distance of $6.19 \mathrm{cM}$ between neighbouring markers and an average number of 9.18 markers in each linkage group and occupying approximately $43.37 \%$ of the total cotton genome. The largest linkage group contained 33 markers, while the smallest one had only 2 markers. Thirty-five linkage groups were assigned to 23 chromosomes, among which 11 were assigned to A genome and 12 were assigned to $\mathrm{D}$ genome. 
Table 2 Correlation coefficients and significances among yield and fiber qualities in different generations

\begin{tabular}{|c|c|c|c|c|c|c|c|}
\hline Trait & Gen. & $\mathrm{FL}$ & FS & $\mathrm{FM}$ & $\mathrm{FE}$ & $\mathrm{FU}$ & BW \\
\hline \multirow[t]{4}{*}{$\overline{F S}$} & $F_{2}$ & $0.375^{* *}$ & & & & & \\
\hline & $\mathrm{F}_{2: 3}$ & $0.495^{* *}$ & & & & & \\
\hline & $F_{2: 4}$ & $0.611^{* *}$ & & & & & \\
\hline & $F_{2: 5}$ & $0.454^{* *}$ & & & & & \\
\hline \multirow[t]{4}{*}{ FM } & $\mathrm{F}_{2}$ & $-0.136^{*}$ & $0.130^{*}$ & & & & \\
\hline & $\mathrm{F}_{2: 3}$ & $-0.390^{* *}$ & $-0.433^{* *}$ & & & & \\
\hline & $F_{2: 4}$ & $-0.402^{* *}$ & $-0.244^{* *}$ & & & & \\
\hline & $F_{2: 5}$ & $-0.309^{* *}$ & $-0.300^{* *}$ & & & & \\
\hline \multirow[t]{4}{*}{$\mathrm{FE}$} & $\mathrm{F}_{2}$ & $0.443^{* *}$ & $0.687^{* *}$ & $0.309^{* *}$ & & & \\
\hline & $F_{2: 3}$ & $-0.437^{* *}$ & $-0.666^{* *}$ & 0.04 & & & \\
\hline & $\mathrm{F}_{2: 4}$ & $-0.722^{* *}$ & $-0.689^{* *}$ & $0.155^{*}$ & & & \\
\hline & $F_{2: 5}$ & $0.672^{* *}$ & $0.586^{* *}$ & 0.038 & & & \\
\hline \multirow[t]{4}{*}{$\mathrm{FU}$} & $\mathrm{F}_{2}$ & $0.213^{* *}$ & $0.434^{* *}$ & $0.350^{* *}$ & $0.357^{* *}$ & & \\
\hline & $F_{2: 3}$ & $0.247^{* *}$ & $0.247^{* *}$ & -0.064 & $-0.331^{* *}$ & & \\
\hline & $\mathrm{F}_{2: 4}$ & $0.151^{*}$ & $0.128^{*}$ & 0.041 & $-0.238^{* *}$ & & \\
\hline & $F_{2: 5}$ & $0.139^{*}$ & $0.149^{*}$ & $0.132^{*}$ & $0.369^{* *}$ & & \\
\hline \multirow[t]{4}{*}{ BW } & $F_{2}$ & $0.250^{* *}$ & $0.204^{* *}$ & $0.466^{* *}$ & $0.240^{* *}$ & $0.420^{* *}$ & \\
\hline & $F_{2: 3}$ & 0.007 & 0.098 & 0.026 & -0.051 & 0.084 & \\
\hline & $F_{2: 4}$ & 0.031 & 0.037 & $0.289^{* *}$ & -0.107 & 0.033 & \\
\hline & $\mathrm{F}_{2: 5}$ & 0.037 & $0.164^{* *}$ & 0.112 & 0.07 & -0.001 & \\
\hline \multirow[t]{4}{*}{$L P$} & $\mathrm{~F}_{2}$ & 0.028 & 0.048 & 0.058 & -0.051 & $0.253^{* *}$ & -0.078 \\
\hline & $\mathrm{F}_{2: 3}$ & $-0.242^{* *}$ & $-0.412^{* *}$ & $0.299^{* *}$ & $0.286^{* *}$ & -0.036 & $-0.350^{* *}$ \\
\hline & $\mathrm{F}_{2: 4}$ & $-0.414^{* *}$ & $-0.412^{* *}$ & $0.479^{* *}$ & $0.304^{* *}$ & 0.003 & $-0.175^{* *}$ \\
\hline & $F_{2: 5}$ & $-0.214^{* *}$ & $-0.405^{* *}$ & $0.379^{* *}$ & $-0.191^{* *}$ & 0.049 & $-0.240^{* *}$ \\
\hline
\end{tabular}

*, ** Correlation is significant at 0.05 and 0.01 probability levels, respectively

\section{QTL mapping for fiber quality traits}

In total, we identified 74 additive QTLs for fiber quality, including 21 stable and 23 major QTLs. In addition, the number of QTLs for the 5 fiber traits varied from 10 to 20, with 20 for FL, 15 for FE, 17 for FS, 10 for FU and 12 for FM (Fig. 1, Additional file 2 Tables S2 and Additional file 3 Table S3). The total number of QTLs in At subgenome was 25, and the other 49 QTLs were located in Dt subgenome. The highest number of QTLs (10) was found on chromosome D5 (chr.19); but only 2 QTL was found on chr.A5. Line 901-001 conferred positive additive alleles for 31 QTLs, and sGK156 conferred positive additive alleles for 43 QTLs.Twenty QTLs for FL were detected on 14 chromosomes, including chromosome 1, 2, 3, 7, 9, 10, 14, 15, 16, 17, $18,19,20$ and 25. Of the total QTLs, 5 were detected in at least 2 generations and regarded as stable, and 8 explained more than $10 \%$ of the PV. Four QTLs (qFL-C2-1, qFL-C9$1, \mathrm{qFL}-\mathrm{C} 19-1$ and $\mathrm{qFL}-\mathrm{C} 20-2)$ were detected in 2 generations, 3 of which (except for qFL-C9-1) were major. The additive effects of qFL-C2-1, qFL-C9-1 and qFL-C19-1 varied from $-0.4829 \mathrm{~mm}$ to $-0.6857 \mathrm{~mm}$, from -0.0285 $\mathrm{mm}$ to $-0.0517 \mathrm{~mm}$ and from $-0.4246 \mathrm{~mm}$ to -0.7579 $\mathrm{mm}$, with PVs explained 10.48\% 13.27\%, 2.69\% 4.67\% and $11.04 \% \sim 1.87 \%$, respectively. FL was increased by the sGK156 allele. The additive effect of qFL-C20-2 varied from $0.3835 \mathrm{~mm}$ to $0.3980 \mathrm{~mm}$, with a PV explained $10.29 \%$ 14.09\% FL was increased by the 901-001 allele. One QTL (qFL-C15-2) was mapped in the same interval of SWU11632a-NAU3353 in $\mathrm{F}_{2}, \mathrm{~F}_{2: 3}$ and $\mathrm{F}_{2: 4}$ generations, with additive effect from $-0.1828 \mathrm{~mm}$ to $-0.3553 \mathrm{~mm}$ and a range of PV explained from $4.70 \%$ to $11.27 \% \mathrm{FL}$ was increased by the sGK156 allele. A total of 17 QTLs for FS were found on 12 chromosomes, including chromosomes $5,7,9,11,12,14,16,19,20,24,25$ and 26. Among these 17 QTLs, only 3 were stably detected in multiple generations, and 5 explained more than $10 \%$ of the observed PV. qFS-C16-2 was mapped in the similar interval of CCR000629-SWU10645 in $\mathrm{F}_{2}, \mathrm{~F}_{2: 3}$ and $\mathrm{F}_{2: 5}$ generations, with additive effects from $-0.0312 \mathrm{cN} \cdot \mathrm{tex}^{-1}$ to $-0.3636 \mathrm{cN} \cdot \mathrm{tex}^{-1}$. FS was increased by the sGK156 allele. Of other 2 stable QTLs, qFS-C19-1 was a major QTL detected in $F_{2: 4}$ and $F_{2: 5}$ generation, with a PV explained from $13.73 \%$ to $13.93 \%$ and additive effect from $-0.5746 \mathrm{cN} \cdot$ tex $^{-1}$ to $-0.7296 \mathrm{cN} \cdot$ tex $^{-1}$; FS was increased by the sGK156 allele. qFS-C24-1 explained $1.01 \%$ and $4.89 \%$ of $\mathrm{PV}$ in $\mathrm{F}_{2}$ and $\mathrm{F}_{2: 3}$ generations, respectively, and the favorable allele came from sGK156. Twelve QTLs for FM were detected on chromosome 3, 5, 7, 14, 15, 16, 17, $19,20,24$ and 25. Five QTLs were stably detected in two generations, and only 2 explained more than $10 \%$ of the observed PV. qFM-C3-1 explained $5.76 \%$ and $7.90 \%$ of $\mathrm{PV}$ in the $\mathrm{F}_{2: 4}$ and $\mathrm{F}_{2: 5}$ generations, respectively. qFMC16-1 was detected in $\mathrm{F}_{2: 3}$ and $\mathrm{F}_{2: 4}$ generations, with a PV of 2.25\% 4.54\%. The favorable alleles for these QTLs that increased FM were from 901-001. The QTLs qFMC7-1, qFM-C14-1 and qFM-C17-1, with a negative additive effect, were detected in 2 generations, indicating that the sGK156 allele increased FM. Ten QTLs for FU were detected on chromosomes 1, 7, 8, 16, 19 and 24. Four QTLs (qFU-C7-2, qFU-C19-1, qFU-C19-3 and qFUC24-2) were identified in two generations, and 3 (qFUC1-1, qFU-C7-1 and qFU-C8-1) explained more than $10 \%$ of the observed PV. qFU-C7-2, qFU-C19-1, and qFU-C19-3 were detected as having a negative additive effect, indicating that the sGK156 allele increased FU. qFU-C24-2 contributed $1.51 \%$ and $3.67 \%$ of PV in the $\mathrm{F}_{2: 4}$ and $\mathrm{F}_{2: 5}$, respectively, and the favorable allele came from 901-001. Fifteen QTLs for FE were detected on 10 chromosomes, including chromosome $2,9,10,14,15,16,18$, 19, 24 and 25. Four QTLs (qFE-C2-1, qFE-C15-2, qFE$\mathrm{C} 18-1$ and $\mathrm{qFE}-\mathrm{C} 25-1)$ were detected in 2 generations, and three explained more than $10 \%$ of PV. qFE-C15-2 explained $7.66 \%$ and $14.3 \%$ of PVs in $\mathrm{F}_{2}$ and $\mathrm{F}_{2: 4}$ generations, respectively. qFE-C18-1 explained $11.25 \%$ and $15.15 \%$ of 


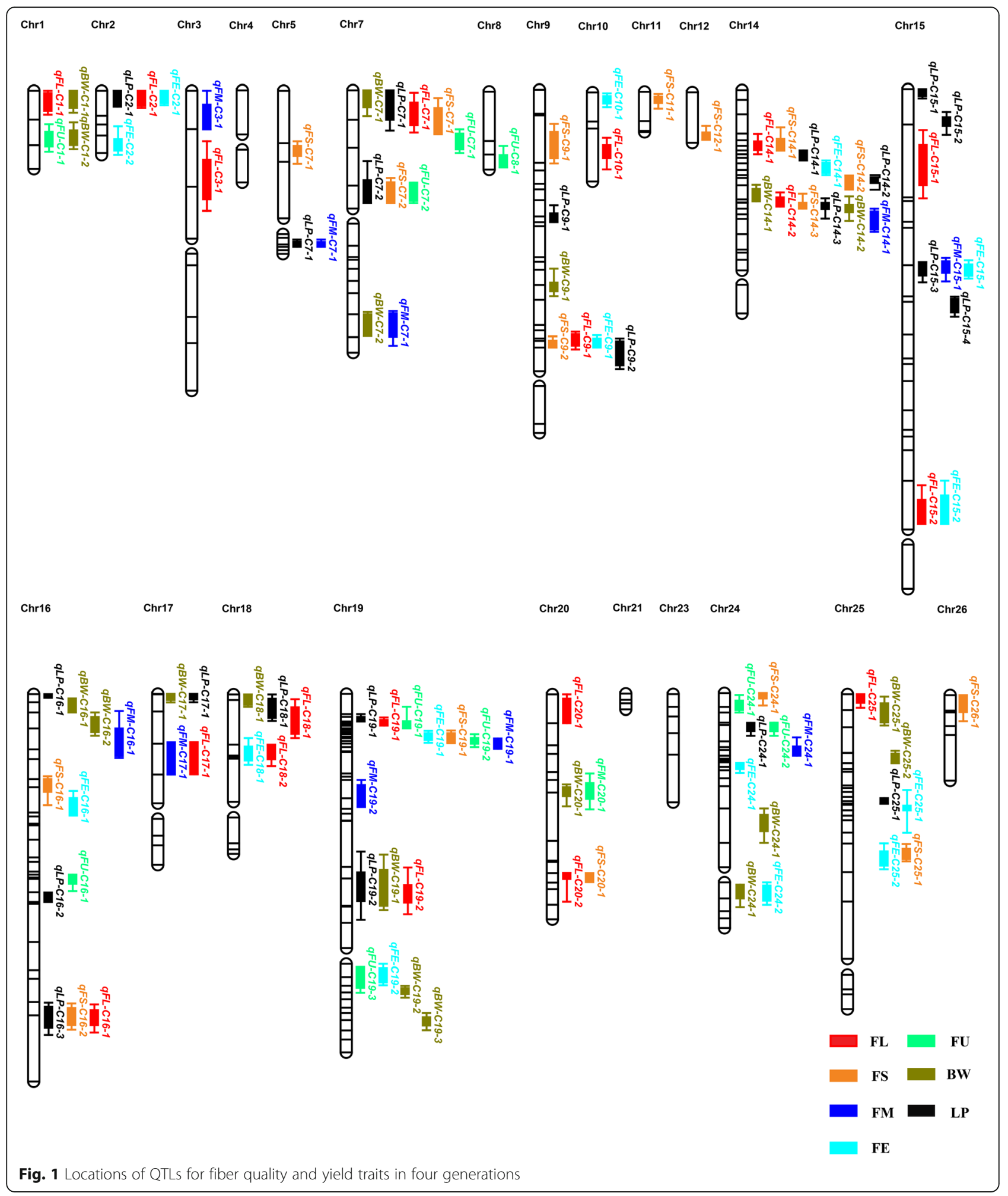

PVs in $F_{2}$ and $F_{2: 4}$ generations, respectively. qFE-C25-1 was detected in $\mathrm{F}_{2: 3}$ and $\mathrm{F}_{2: 4}$ generations with a range of PV from $12.05 \%$ to $25.41 \%$. The favorable allele for these three QTLs were from sGK156. Other two major QTLs, qFE-C9-1 and qFE-C19-1, were detected in 1 generation and explained $11.38 \%$ and $11.85 \%$ of PVs, respectively. The favorable allele for these QTLs were from 901-001. 


\section{QTL mapping for yield-related traits}

Forty-one QTLs for yield traits were identified in the four generations with 19 QTLs for BW and 22 QTLs for LP (Fig. 1, Additional file 2 Tables S2 and Additional file 4 Table S4), including 14 stable and 9 major QTLs. Thirteen QTLs were located in At sub-genome, and other 28 QTLs were located in Dt sub-genome. The favorable additive alleles for 20 QTLs originated from 901-001, other alleles for 21 QTLs originated from sGK156.

Nineteen QTLs for BW were detected on 11 choromosomes, 5 of which (qBW-C14-2, qBW-C17-1, qBWC19-2, qBW-C19-3 and qBW-C24-2) were detected in at least 2 generations. $\mathrm{qBW}-\mathrm{C} 17-1$ was identified in 3 generations $\left(\mathrm{F}_{2}, \mathrm{~F}_{2: 3}\right.$ and $\left.\mathrm{F}_{2: 5}\right)$ with a range of $\mathrm{PV}$ explained from $1.84 \%$ to $8.47 \%$, and 901-001 allele increased boll weight by $0.1020 \mathrm{~g} \sim 0.2720 \mathrm{~g}$. Three QTLs (qBW-C142, qBW-C19-2 and qBW-C24-2) were detected with a negative additive effect, indicating that sGK156 allele increased BW. qBW-C19-3 explained $1.18 \%$ to $2.74 \%$ of the observed PV, and the favorable allele was from 901-001.

Twenty-two QTLs for LP were identified on 12 chromosomes, including chromosome 2, 5, 7, 9, 14, 15, 16, $17,18,19,24$ and 25, and these QTLs explained 1.03\% to $27.8 \%$ of the observed PV. Nine of these QTLs were detected in at least 2 generations, and 5 explained more than $10 \%$ of the observed PV. qLP-C16-3 was detected in 3 generations $\left(F_{2: 3}, F_{2: 4}\right.$ and $\left.F_{2: 5}\right)$, explaining from $6.04 \%$ to $11.97 \%$ of the observed PV, with a positive additive effect of 0.4123 to 0.8676 , indicating that 901001 allele increased LP. QTL qLP-C17-1 was also detected in 3 generations $\left(\mathrm{F}_{2}, \mathrm{~F}_{2: 3}\right.$ and $\left.\mathrm{F}_{2: 5}\right)$, but its additive effect was negative. The other 7 stable QTLs (qLP-C7-1, qLP-C7-2, qLP-C14-1, qLP-C14-2, qLP-C14-3, qLPC15-2 and qLP-C19-2) were detected in 2 generations. The 901-001 alleles increased LP for qLP-C7-1, qLPC14-1 and qLP-C14-2, and the favorable allele for qLPC7-2, qLP-C14-3, qLP-C15-2, and qLP-C19-2 was from sGK156.

\section{QTL clusters}

A total of 15 QTL clusters were found on 11 chromosomes, and these involved more than half of the QTLs (60/115, 52.17\%), including 12 FL-QTLs $(60.00 \%), 9$ FSQTLs (52.94\%), 5 FM-QTLs (41.67\%), 5 FU-QTLs (50.00\%), 8 FE-QTLs (53.33\%), 5 BW-QTLs (26.32\%), and 16 LP- QTLs (72.73\%) (Additional file 5 Tables S5 and Additional file 6 Table S6). Detailed information on the clusters and their distribution in the genome are included in Additional file 7 Table S7 and Fig. 1.

The cluster c1-cluster-1 was in the region from 4 to $24 \mathrm{cM}$ on chr.1, and 4. QTLs were detected for 2 fiber quality traits (qFL-C1-1 and $\mathrm{qFU}-\mathrm{C} 1-1$ ) and 2 yield-related traits (qBW-C1-1 and $\mathrm{qBW}-\mathrm{C} 1-2)$, indicating that the additive effects increased $\mathrm{BW}$ and $\mathrm{FU}$, and decreased FL. Three QTLs (qFL-C2-1, qFE-C2-1 and qLP-C2-1) were clustered in c2-cluster-1 from 0 to $7 \mathrm{cM}$ on chr.2, which increased LP, and decreased FL and FE. Additionally, the QTLs qFL-C2-1 and qFE-C2-1 were detected stably in two generations. Chr. 7 contained 2 clusters (c7-cluster-1 and c7-cluster-2), in which c7-cluster-1, in the region from 0 to $18 \mathrm{cM}$, influenced different traits, including FL (qFL-C7-1), FS (qFS-C7-1), LP (qLP-C71) and $\mathrm{BW}(\mathrm{qBW}-\mathrm{C} 7-1)$, while the QTL $\mathrm{qLP}-\mathrm{C} 7-1$ was detected stably in two generations. In regards to the additive effects, this QTL-cluster could increase FL, FS and LP, and decrease BW. Three QTLs (qFS-C7-2, qFU-C7-2 and qLP-C7-2) were clustered in c7-cluster2 from 38 to $47 \mathrm{cM}$ on chr.7, indicating that this cluster could increase FS, and decrease FU and LP. Additionally, the QTLs qFU-C7-2 and qLP-C7-2 were detected stably in two generations. c9-cluster-1 in the region from 25 to $43 \mathrm{cM}$ on chr.9 harbored 4 QTLs (qFL-C9-1, qFS-C9-2, qFE-C9-1 and qLP-C9-2), which increased FE and LP, and decrease FL and FS. Additionally, the QTL qFL-C9-1 was detected stably in two generations. Chr.14 contained 2 clusters (c14cluster-1 and c14-cluster-2), in which c14-cluster-1, in the region from 25 to $43 \mathrm{cM}$ influenced different traits, including FL (qFL-C14-1), FS (qFS-C14-1 and qFS-C14-2), FE (qFE-C14-1), LP (qLP-C14-1 and qLP-C14-2), while QTLs qLP-C14-1 and qLP-C14-2 were detected stably in two generations. In regards to the additive effects, this QTL cluster could increase FE and LP, and decrease FL and FS. Cluster c14-cluster-2 $(48-60 \mathrm{cM})$ harbored 6 QTLs (qFL-C14-2, qFSC14-3, qFM-C14-1, qBW-C14-1, qLP-C14-2 and qLP-C14-3) that influenced 5 traits, increasing FL and decreasing FS, FM, BW and LP. Additionally, the QTLs qFM-C14-1 and qLP-C14-3 were detected stably in two generations. In the region from 80 to 101 cM on chr.15, c15-cluster-1 harbored 4 QTLs (qFMC15-1, qFE-C15-1, qLP-C15-3 and qLP-C15-4), which increased FE and decreased FM and LP. Cluster c16-cluster-1 $(146-150 \mathrm{cM})$ harbored 3 QTLs (qFL-C16-1, qFS-C16-2 and qLP-C16-3), which increased LP, and decrease FL and FS. Additionally, QTL qLP-C16-3 was detected stably in two generations. Cluster c18-cluster-1, in the region from 8 to $28 \mathrm{cM}$ on chr.18, harbored 4 QTLs (qFL-C18-1, qFL-C18-2, qFE-C18-1 and qLP-C18-1), which increased LP, and decreased FL and FE. Chr. 19 contained 2 clusters (c19-cluster-1 and c19-cluster-2). Seven QTLs (qFL-C19-1, qFS-C19-1, qFM-C19-1, qFU-C19-1, qFU-C19-2, qFE-C19-1 and qLP-C191 ) were detected in the region from 12 to $24 \mathrm{cM}$ on chr.19 (c19-cluster-1), and 3 of these QTLs, namely, qFL-C19-1, qFS-C19-1 and qFU-C19-1, were stable. 
The additive effects on FM and FE were positive, and negative effect for fiber length, fiber strength, fiber uniformity and lint percentage. The cluster c19-cluster-2 (84-94 cM) harbored 3 QTL (qFL-C19-2, qBW-C19-1 and qLP-C19-2) that influenced 3 traits, increasing FL and BW, and decreasing LP. Additionally, QTL qLP-C192 was detected stably in two generations. Cluster c24-cluster-1, in the region from 14 to $27 \mathrm{cM}$, harbored $3 \mathrm{QTL}$ (qFU-C24-2, qFM-C24-1 and qLP-C24-1) that influenced 3 traits, increasing FU and FM and decreasing LP. Additionally, QTL qFU-C24-2 was detected stably in two generations. Chr.25 contained 2 clusters (c25-cluster-1 and c25-cluster-2). Cluster c25-cluster-1 in the region from 45 to $61 \mathrm{cM}$, contained 3 QTLs (qFE-C25-1, qFMC25-1 and qLP-C25-1), QTL qFE-C25-1 was detected stably in two generations, and additive genetic effects of these QTLs were negative. Cluster c25-cluster-2 (74-77 cM) which harbored 3 QTL (qFL-C25-2, qFS-C25-1 and qFE-C25-2) were found to be associated with marker COT002, and accounted to increased FE and decreased FL and FS.

In brief, the main fiber quality and yield traits were negatively correlated in most of clusters (C1-cluster-1, C2-cluster-1, C7-cluster-2, C9-cluster-1, C14-cluster-1, C14-cluster-2, C16-cluster-1, C18-cluster-1, C19-cluster2 and C24-cluster-1).

\section{Discussion}

\section{Mapping population types for MAS breeding}

Breeders have long recognized the significant negative association between lint yield and fiber quality. Although conventional breeding has played a vital role in the genetic improvement of lint yield and fiber quality in Upland cotton, the achievement and progress have been slow (Zhang et al. 2012). The utilization of marker-assisted selection (MAS) makes it possible for plant breeders to identify rapid and precise approaches for improving conventional selection schemes (Moose and Mumm 2008; Tanksley and Hewitt 1988).

To implement MAS in cotton breeding, first, it is imperative to identify many stable and major QTLs for cotton yield and fiber quality. In previous years, many studies on genetic map construction and QTL identification were conducted. However, populations was mainly developed for basic studies (Rong et al. 2004; Shen et al. 2007; Sun et al. 2012; Ning et al. 2014; Said et al. 2015; Jamshed et al. 2016; Shang et al. 2015; Tang et al. 2015; Zhai et al. 2016; Liu et al. 2017). In our research, the population was developed from hybrid CCRI 70 with its parents, which is a nationally authorized cotton variety with excellent fiber quality. The use of this resource would facilitate combining the results of QTL identification and breeding and could provide information on fiber quality and yield traits improvements in cotton.

\section{Comparison of QTL with the previous reports}

Currently, different mapping populations and markers were applied in QTL localization, and thus making it difficult to compare with different studies. We identified 115 QTLs related to fiber quality and yield traits in the populations of CCRI 70 and compared with those detected in previous relevant studies (Chen et al. 2008; Jamshed et al. 2016; Qin et al. 2008; Shen et al. 2005; Sun et al. 2012; Shao et al. 2014; Shang et al. 2015; Tang et al.2015; Wang et al. 2008, 2010; Yang et al. 2007; Yu et al. 2013; Zhang et al. 2008, 2012; Zhai et al. 2016; Liu et al. 2017), and 25 QTLs were found to be consistent with those in previous studies.

Among the QTLs for FL, qFL-C3-1 might be the same as qFL-2-1(on chr.3) (Wang et al. 2010), with the closely anchored marker CGR6528. qFL-C7-1 was mapped to chr.7 same as FL-C7-1 (Sun et al. 2012), qFL-7-1a (Shen et al. 2005), qFL-C7-2 (Jamshed et al. 2016), based on common markers NAU1048 and NAU1045. qFL-C14-1 was same as qFL14.1 (Liu et al. 2017) with common markers SWU14599, SWU14643, CGR5258 and SWU14616. qFL-C20-2 might be the same as FL-20-3 (Zhai et al. 2016), with the closely anchored marker CGR5565. Shao et al. (2014) reported QTL qFL15.1 and Tang et al. (2015) reported QTL qFL16.1, which were similar to qFL-C15-2 and qFL-C16-1 in our study.

Among QTLs for FS, qFS-C7-2, which was mapped on chr.7, sharing similar linked markers NAU1048, NAU1045 and MUSS004 with QTLs detected in previous reports (Chen et al. 2008; Sun et al. 2012; Jamshed et al. 2016). qFS-C14-1 was same as qFS14.2 in Liu et al. (2017), based on common markers SWU14599, SWU14643, CGR5258 and SWU14616. qFS-C20-1 might be the same as FS-20-3 (Zhai et al. 2016), with the closely anchored marker CGR5565. qFS-C25-1 was same as qFS25.1 in Shao et al. (2014), with common linked markers CGR6584, COT002 and CGR5525. qFS-C16-2 was similar to qFS16.1 in Tang et al. (2015).

Among QTLs for FM, qFM-C5-1 was the same as qFMIC-A5-1 in Yang et al. (2007), based on common markers NAU4031, CGR5077 and NAU1200. qFM-C7-1 was assigned to same chromosome as a QTL linked to the common marker BNL1694 (Sun et al. 2012). qFM-C19-1 was the same as qFM-C19-1, with the common markers NAU0797, NAU1042 and HAU0878 (Shang et al. 2015). Among QTLs for FU, qFU-C7-1 was the same as a QTL linked to the common marker NAU1085, NAU1048 and MUSS004 (Sun et al. 2012). qFU-C16-1 was assigned to the same chromosome as a QTL linked to the common marker NAU5120 (Zhang et al. 2012). qFE-C14-1 was same as qFE14.1 (Liu et al. 2017), with common markers SWU14599, SWU14643, CGR5258, SWU14616. qFE-C251 and qFE-C25-2 may be the same as qFE-C25- 1 and 
qFE-C25-2 (Jamshed et al. 2016), based on the common markers BNL0584, SWU19042, CGR6584, DPL0375, CGR5525 and COT002, as well as qFE-C25-1 and qFEC25-2, with the closely anchored markers CGR5525 and COT002 in both studies, respectively. A QTL (qFE-C19-1) for FE located on chr.19 was the same as qFE-C19-1 in the previous study (Sun et al. 2012).

Among QTLs for BW, one QTL (qBW-C7-1) located on chr.7 was the same as qBS-A7-1 in Wang et al. (2008), based on shared linked marker NAU1085; qBWC7-2 was detected in the same marker intervals as a QTL linked to marker BNL1694 in Chen et al. (2008), and $\mathrm{Yu}$ et al. (2013). Among QTLs for LP, qLP-C7-1 and qLP-C7-2, located on chr.7, were the same as qLP5-2 (on chr.7) and qLP-5-2 in Zhang et al. (2008), based on shared markers NAU1085, NAU1048 and MUSS004. qLP-C14-1 was same as qLP14.1 (Liu et al. 2017), with common markers SWU14599, SWU14643, CGR5258 and SWU14616.

A total of 25 QTLs were found to be consistent with previous studies, and 35 were detected stably in multiple generations. Further analysis showed that 7 of the 25 QTLs were detected stably in multiple generations. Thus, 53 QTLs were detected stably in multiple generations or different genetic backgrounds and thus could be considered to use in MAS. Special attentions should be paid to these stable QTLs and to those detected in previous studies, because stable QTLs add valuable information for further QTL fine mapping and gene positional cloning for fiber quality and yield-related traits genetic detection and providing useful markers for further molecular breeding.

\section{QTL clusters in cotton genome}

The phenomenon of QTL clustering has been reported in cotton (Shen et al. 2007; Wan et al. 2007; Qin et al. 2008; Yu et al. 2013; Zhang et al. 2009; Tang et al. 2015; Wang et al. 2015; Ning et al. 2014; Said et al. 2013, 2015; Nie et al. 2016; Jamshed et al. 2016; Zhai et al. 2016; Liu et al. 2017; Guo et al. 2018). This phenomenon was also observed in our results. In this study, a total of 15 QTL clusters were identified to affect three or more different fiber quality or yield-related traits on chromosomes $1,2,7,9,14,15,16,18,19,24$ and 25 , which indicated that fiber quality and yield traits showed significantly complicated correlations (Rong et al. 2004; Shen et al. 2005; Shang et al. 2015; Zhai et al. 2016; Liu et al. 2017). The significantly positive correlation between FL and FS was explained by their co-localized QTLs with the same positive or negative additive effects (c7-cluster-1, c9-cluster-1, c16-cluster-1, c19-cluster-1 and c25-cluster-2). The significantly negative correlation between FM and FS or FL may be explained by the clusters of QTLs with opposite additive effects (positive and negative) (c14-cluster-2 and c19-cluster-1). In addition, the significantly negative correlation between LP and FL, and FS may be explained by their coinciding QTLs with opposite additive effects (c2-cluster-1, c7cluster-2, c9-cluster-1, c14-cluster-1, c14-cluster-2, c16-cluster-1, c18-cluster-1 and c19-cluster-2). Notably, the regions on chr.7 (c7-cluster-1) and chr.19 (c19-cluster-1) were strongly related to fiber quality and yield traits, which revealed that quality and yield traits can be improved synchronously.

Most of the clusters showed opposite additive genetic effects for fiber quality and yield related traits in previous reports. Wang et al. (2013) reported that a QTL-rich region on chr.7 was associated with FL, FS and LP, and the direction of genetic effects of QTLs on FL and FS was positive, but the direction was the opposite for fiber quality traits and LP. The NAU3308-NAU4024 interval on D2 harbored seven significant QTLs related to FL, FS, FE LP, LY, SI and NB, which showed opposite additive effects on fiber quality and yield related traits (Qin et al. 2008). Wan et al. (2007) reported that a QTL-cluster in the $t_{1}$ locus region on chr.6 increased FL, FS, FE and FU, and decrease LP. Wang et al. (2015) reported two important clusters in the region from 70 to $86 \mathrm{cM}$ on LG1-chr1/15 and $18-37 \mathrm{cM}$ on chr.21. The cluster on LG1-chr1/15 were correlated with FS, FM, FE and LP and the cluster on chr. 21 were correlated with FL, FS, LP, SCW and CI, the additive effect for these QTLs of traits (except FE) were positive, which revealed that fiber quality and yield traits could be improved synchronously.

In conclusion, the clustering of QTLs for fiber quality and yield traits further proved the strong correlation among fiber qualities and yield traits (Qin et al. 2008; Wang et al. 2013). To improve fiber quality and yield potential at the same time, fine mapping of these QTLrich intervals on specific chromosomes are necessary for the future application in MAS and gene cloning (Guo et al. 2018; Zhai et al. 2016).

\section{Stability of major QTLs across multiple generations}

In recent years, with the development and improvement of molecular quantitative genetics and relevant analysis software, increasing numbers of major QTLs that can be used for MAS have been accurately located (Shang et al. 2015). QTLs that can be simultaneously detected in different generations (or different environments) were stable and useful (Su et al. 2010; Guo et al. 2005). The aim of QTL analysis by molecular markers is to inform MAS; thus, the stability and usefulness of QTLs are important (Wang et al. 2011). In this study, 35 QTLs were detected in at least 2 generations. Among these, which were detected stably in multiple generations, 13 QTLs (qFL-C2-1, qFL-C15-2, qFL-C19-1, qFL-C20-2, qFSC19-1, qFM-C17-1, qFE-C15-2, qFE-C18-1, qFE-C25- 
1, qBW-C14-2, qBW-C19-2, qLP-C14-2 and qLP-163) were major QTLs explaining most PV on average (average PV explained $>10 \%$ ). Five of the 35 QTLs were detected in 3 generations, including 1 QTL for FL (qFL15-1), 1 QTL for FS (qFS-16-2,), 1 QTL for BW (qBW17-1) and 2 QTL for LP (qLP-16-3 and qLP-17-1). Some of these multiple generationally stable and major QTLs belonged to clusters, as follows: qFL-C2-1, qLPC14-2, qFS-16-2, qLP-16-3, qFL-C19-1, qFS-C19-1 and qFE-C25-1, which belong to C2-cluster-1, C14-cluster-1, C16-cluster-1, C19-cluster-1 and C25-cluster-1, respectively. Furthermore, some of these QTLs were also reported in other populations, including qLP-C14-2 (Liu et al. 2017), qFL-C15-2 (Shao et al. 2014), qFS-16-2 (Tang et al. 2015) and qFL-C20-2 (Zhai et al. 2016).

The stability of these QTLs across generations or populations and outstanding chromosomal regions motivates further interests in study, and the alleles underlying them are valuable candidate genes either for implementation in MAS or for studies of the molecular mechanism of fiber quality and yield-related traits.

\section{Conclusions}

QTL mapping was used to analyze molecular genetic mechanism of fiber quality and yield component traits using a series of generations $\left(\mathrm{F}_{2}, \mathrm{~F}_{2: 3}, \mathrm{~F}_{2: 4}\right.$ and $\left.\mathrm{F}_{2: 5}\right)$ that constructed from CCRI 70. Fiber quality and yield-related traits showed significant and complex correlations. A total of 115 QTLs for fiber quality and yield-related traits were detected. Of these QTLs, 53 were detected stably in multiple generations or different genetic backgrounds, which could indicate their potential use in MAS. In addition, 15 QTL clusters were found in 11 chromosomal segments. Determining the locations of these clusters will be beneficial for MAS and breeding programs that focused on fiber quality and yield related traits.

\section{Additional files}

Additional file 1: Table S1. Correlation coefficients of same trait among different generations. (DOCX $15 \mathrm{~kb}$ )

Additional file 2: Table S2. Distribution of fiber quality and yield QTLS across the cotton genome. (DOCX $15 \mathrm{~kb}$ )

Additional file 3: Table S3. QTLs of fiber traits detected by CIM in four generations. (DOCX $44 \mathrm{~kb}$ )

Additional file 4: Table S4. QTLs of yield trait detected by CIM in four generations. (DOCX $23 \mathrm{~kb}$ )

Additional file 5: Table S5. Distribution of fiber quality and yield QTLS across the clusters. (DOCX $14 \mathrm{~kb}$ )

Additional file 6: Table S6. Total QTLs and coinciding QTLs for the fiber quality and yield related traits. (DOCX $14 \mathrm{~kb}$ )

Additional file 7: Table S7. Distribution of clusters over genome. (DOCX $17 \mathrm{~kb}$ )

Additional file 8: Table S8. The genetic positions of the markers on the map. (XLSX $17 \mathrm{~kb}$ )

\section{Abbreviations}

BW: Boll weight; cM: centimorgans; FE: Fiber elongation; FL: Fiber length; FM: Fiber micronaire; FS: Fiber strength; FU: Fiber uniformity; HVICC: International high-volume instrument calibration cotton; LOD: Logarithm of odds; LP: Lint percentage; MAS: Marker assisted selection; PV: Phenotypic variance; QTL: Quantitative trait locus; SSR: Simple sequence repeat

\section{Acknowledgements}

Not applicable.

\section{Authors' contributions}

Yuan YL and Wu YX conceived and designed the study. Deng XY performed the experiments, data analysis, and wrote the manuscript. Gong JW and Liu AY constructed and investigated phenotypes of the 4 generations. Shi YZ, Gong WK, Ge Q, Li JW and Shang HH prepared the materials and performed the DNA extractions. Yuan YL revised the manuscript. All the authors read and approved the final manuscript.

\section{Funding}

This work was supported by the National Natural Science Foundation of China (31371668) and the National Agricultural Science and Technology Innovation project for CAAS (CAAS-ASTIP-2016-ICR).

\section{Availability of data and materials}

The datasets generated and analysed during the current study are available from the corresponding author on reasonable request.

Ethics approval and consent to participate

Not applicable.

Consent for publication

Not applicable.

\section{Competing interests}

The authors declare that they have no competing interests.

Received: 28 March 2019 Accepted: 19 July 2019

Published online: 04 September 2019

\section{References}

Ademe MS, He SP, Pan ZE, et al. Association mapping analysis of fiber yield and quality traits in upland cotton (Gossypium hirsutum L.). Mol Gen Genomics. 2017;292:1267-80. https://doi.org/10.1007/s00438-017-1346-9.

Chen L, Zhang ZS, Hu MC, et al. Genetic linkage map construction and QTL mapping for yield and fiber quality in upland cotton (Gossypium hirsutum L.). Acta Agron Sin. 2008:34:1199-205. https://doi.org/10.3724/SP.J.1006.2008.01199.

Guo LX, Shi YZ, Gong JW, et al. Genetic analysis of the fiber quality and yield traits in G. hirsutum background using chromosome segments substitution lines (CSSLs) from Gossypium barbadense. Euphytica. 2018;214(82). https://doi. org/10.1007/s10681-018-2158-7.

Guo WZ, Cai CP, Wang CB, et al. A microsatellite-based, gene-rich linkage map reveals genome structure, function, and evolution in Gossypium. Genetics. 2007:176:527-41. https://doi.org/10.1534/genetics.107.070375.

Guo WZ, Zhang TZ, Ding YZ, et al. Molecular marker assisted selection and pyramiding of two QTLs for fiber strength in upland cotton. Acta Genet Sin. 2005;32:1275-85. https://doi.org/10.1016/j.tplants.2005.10.001.

Jamshed M, Jia F, Gong JW, et al. Identification of stable quantitative trait loc (QTLs) for fiber quality traits across multiple environments in Gossypium hirsutum recombinant inbred line population. BMC Genomics. 2016;17:1-13. https://doi.org/10.1186/s12864-016-2560-2.

Kosambi DD. The estimation of map distance from recombination values. Ann Eugenics. 1994;12:172-5. https://doi.org/10.1111/j.1469-1809.1943.tb02321.x.

Lacape JM, Gawrysiak G, Tuong-Vi C, et al. Mapping QTLs for traits related to phenology, morphology and yield components in an inter-specific Gossypium hirsutum $\times$ G.barbadense cotton RIL population. Field Crop Res. 2013;144:256-67. https://doi.org/10.1016/j.fcr.2013.01.001.

Lacape JM, Nguyen TB, Thibivilliers S, et al. A combined RFLP-SSR AFLP map of tetraploid cotton based on a Gossypium hirsutum $\times$ Gossypium barbadense backcross population. Genome. 2003;46:612-26. https://doi.org/10.1139/g03-050. 
Liu XY, Teng ZH, Wang JX, et al. Enriching an intraspecific genetic map and identifying QTL for fiber quality and yield component traits across multiple environments in upland cotton (Gossypium hirsutum L.). Mol Gen Genomics. 2017;292:1281-306. https://doi.org/10.1007/s00438-017-1347-8.

McCouch SR, Cho YG, Yano M, et al. Report on QTL nomenclature. Rice Genetics Newsletter. 1997;14:11-3. https://archive.gramene.org/newsletters/rice_ genetics/rgn14/v14p11.html .

Moose SP, Mumm RH. Molecular plant breeding as the foundation for 21st century crop improvement. Plant Physiol. 2008;147:969-77. https://doi.org/1 0.1104/pp.108.118232.

Nie XH, Huang C, You CY, et al. Genome-wide SSR-based association mapping for fiber quality in nation-wide upland cotton inbreed cultivars in China. BMC Genomics. 2016;17:352. https://doi.org/10.1186/s12864-016-2662-X.

Ning ZY, Chen H, Mei HX, et al. Molecular tagging of QTLs for fiber quality and yield in the upland cotton cultivar Acala-Prema. Euphytica. 2014;195:143-56. https://doi.org/10.1007/s10681-013-0990-3.

Paterson AH, Brubaker CL, Wendel JF. A rapid method for extraction of cotton (Gossypium spp.) genomic DNA suitable for RFLP or PCR analysis. Plant Mol Biol Report. 1993;11(2):122-7. https://link.springer.com/article/10.1007\%2 FBF02670470.

Qin HD, Guo WZ, Zhang YM, et al. QTL mapping of yield and fiber traits based on a four-way cross population in Gossypium hirsutum L. Theor Appl Genet. 2008;117:883-94. https://doi.org/10.1007/s00122-008-0828-x.

Rong JK, Abbey C, Bowers JE, et al. A 3347-locus genetic recombination map of sequence-tagged sites reveals features of genome organization, transmission and evolution of cotton (Gossypium). Genetics. 2004;166:389-417. https://doi. org/10.1534/genetics.166.1.389.

Said Jl, Song MZ, Wang HT, et al. A comparative meta-analysis of QTL between intraspecific Gossypium hirsutum and interspecific $G$. hirsutum $\times G$. barbadense populations. Mol Genet Genomics. 2015;290(3):1003-25. https:// doi.org/10.1007/s00438-015-1021-y.

Shang LG, Liang QZ, Wang YM, et al. Identification of stable QTLs controlling fiber traits properties in multi-environment using recombinant inbred lines in upland cotton (Gossypium hirsutum L.). Euphytica. 2015;205(3):877-88. https:// doi.org/10.1007/s10681-015-1434-z.

Shao QS, Zhang FJ, Tang SY, et al. Identifying QTL for fiber quality traits with three upland cotton (Gossypium hirsutum L.) populations. Euphytica. 2014; 198(1):43-58. https://doi.org/10.1007/s10681-014-1082-8.

Shen XL, Guo WZ, Lu QX, et al. Genetic mapping of quantitative trait loci for fiber quality and yield trait by RIL approach in upland cotton. Euphytica. 2007; 155(3):371-80. https://doi.org/10.1007/s10681-006-9338-6.

Shen XL, Guo WZ, Zhu XF, et al. Molecular mapping of QTLs for qualities in three diverse lines in upland cotton using SSR markers. Mol Breed. 2005;15:169-81. https://doi.org/10.1007/s11032-004-4731-0.

Su CF, Lu WF, Zhao TJ, et al. Verification and fine-mapping of QTLs conferring days to flowering in soybean using residual heterozygous lines. Chin Sci Bull. 2010;55(6):499-508. https://doi.org/10.1007/s11434-010-0032-7.

Sun FD, Zhang JH, Wang SF, et al. QTL mapping for fiber quality traits across multiple generations and environments in upland cotton. Mol Breed. 2012; 30(1):569-82. https://doi.org/10.1007/s11032-011-9645-z.

Tang SY, Teng ZH, Zhai TF, et al. Construction of genetic map and QTL analysis of fiber quality traits for upland cotton (Gossypium hirsutum L.). Euphytica. 2015;201:195-213. https://doi.org/10.1007/s10681-014-1189-y.

Tanksley SD, Hewitt J. Use of molecular markers in breeding for soluble solids content in tomato—a re-examination. Theor Appl Genet. 1988;75(5):811-23. https://doi.org/10.1007/bf00265610.

Voorrips RE. MapChart 2.2: software for the graphical presentation of linkage maps and QTLs. J Hered. 2006;93(1):77-8. https://academic.oup.com/jhered/ article/93/1/77/2187477.

Wan Q, Zhang ZS, Hu MC, et al. T1 locus in cotton is the candidate gene affecting lint percentage, fiber quality and spiny bollworm (Earias spp.) resistance. Euphytica. 2007;158(1-2):241-7. https:/doi.org/10.1007/s10681-007-9446-y.

Wang FR, Gong YC, Zhang CY, et al. Genetic effects of introgression genomic components from Sea Island cotton (Gossypium barbadense L.) on fiber related traits in upland cotton (G. hirsutum L.). Euphytica. 2011;181(1):41-53. https://doi.org/10.1007/s10681-011-0378-1.

Wang FR, Xu ZZ, Sun R, et al. Genetic dissection of the introgressive genomic components from Gossypium barbadense $\mathrm{L}$. that contribute to improved fiber quality in Gossypium hirsutum L. Mol Breeding. 2013;32:547-62. https://doi. org/10.1007/s11032-013-9888-y.
Wang HT, Huang C, Guo HL, et al. QTL mapping for fiber and yield traits in upland cotton under multiple environments. PLoS One. 2015;10(6):e0130742. https://doi.org/10.1371/journal.pone.0130742.

Wang PZ, Qin L, Su L, et al. QTL mapping of the partial yield components of main upland cotton cultivars planted in Xinjiang. Sci Agric Sin. 2008;41(10): 2947-56. https://doi.org/10.3864/j.issn.0578-1752.2008.10.006.

Wang S, Basten CJ, Zeng ZB. Windows QTL cartographer 2.5. Raleigh: Department of Statistics: North Carolina State University; 2006. https:// brcwebportal.cos.ncsu.edu/qtlcart/WQTLCart.htm

Wang YQ, Li JW, Shi YZ, et al. Molecular marker of QTL for fiber quality traits in upland cotton with elite fiber quality. Cotton Science. 2010;22(6):533-8.

Xia Z, Zhang X, Liu YY, et al. Major gene identification and quantitative trait locus mapping for yield-related traits in upland cotton (Gossypium hirsutum L.). J Integr Agric. 2014;13(2):299-309. https://doi.org/10.1016/\$2095-3119(13)60508-0.

Yang C, Guo WZ, Zhang TZ. QTL mapping for resistance to Verticillium wilt, fiber quality and yield traits in upland cotton (Gossypium hirsutum L.). Molecular Plant Breeding. 2007:5:797-805

Ye ZW, Deng XY, Shi YZ, et al. Analysis of phenotypic variation in fiber yield and quality in segregative populations of hybrid cotton CCRI 70. Cotton Science. 2016;28:1-10. https://doi.org/10.11963/issn.1002-7807.201601001.

Yu JW, Zhang K, Li SY, et al. Mapping quantitative trait loci for lint yield and fiber quality across environments in a Gossypium hirsutum×Gossypium barbadense backcross inbred line population. Theor Appl Genet. 2013;126:275-87. https://doi.org/10.1007/s00122-012-1980-x.

Yuan YL, Shi YZ, Li JW, et al. Transgenic insect-resistant cotton of high quality cotton-CCRI 70. China Cotton. 2009;36(2):17. https://doi.org/10.3969/j.issn.1 000-632X.2009.02.008.

Zeng ZB. Precision mapping of quantitative trait loci. Genetics. 1994;136:1457-68. https://doi.org/10.1007/s00122-012-2032-2.

Zhai HC, Gong WK, Tan YN, et al. Identification of chromosome segment substitution lines of Gossypium barbadense introgressed in G. hirsutum and quantitative trait locus mapping for fiber quality and yield traits. PLoS One. 2016;11(9):e0159101. https://doi.org/10.1371/journal.pone.0159101.

Zhang J, Wu YT, Guo WZ, et al. Fast screening of microsatellite markers in cotton with PAGE/silver staining. Cotton Science. 2000;12(5):267-9. https://doi.org/1 0.3969/j.issn.1002-7807.2000.05.012.

Zhang JH, Wang SF, Shi YZ, et al. Molecular marker and QTL of yield-related traits in transgenic insect-resistant cotton varieties. Cotton Science. 2008;20:179-85. https://doi.org/10.3969/j.issn.1002-7807.2008.03.004.

Zhang K, Zhang J, Ma J, et al. Genetic mapping and quantitative trait locus analysis of fiber quality traits using a three-parent composite population in upland cotton (Gossypium hirsutum L.). Mol Breed. 2012;29:335-48. https:// doi.org/10.1007/s11032-011-9549-y.

Zhang TZ, Qian N, Zhu XF, et al. Variations and transmission of QTL alleles for yield and fiber qualities in upland cotton cultivars developed in China. PLoS One. 2013;8:e57220. https://doi.org/10.1371/journal.pone.0057220.

Zhang ZS, Hu MC, Zhang J, et al. Construction of a comprehensive PCR based marker linkage map and QTL mapping for fiber quality traits in upland cotton (Gossypium hirsutum L.). Mol Breed. 2009;24:49-61. https://doi.org/10.1 007/s11032-009-9271-1.

Ready to submit your research? Choose BMC and benefit from:

- fast, convenient online submission

- thorough peer review by experienced researchers in your field

- rapid publication on acceptance

- support for research data, including large and complex data types

- gold Open Access which fosters wider collaboration and increased citations

- maximum visibility for your research: over $100 \mathrm{M}$ website views per year

At BMC, research is always in progress.

Learn more biomedcentral.com/submissions 